\title{
LidAR CHARACTERIZATION OF BOUNDARY LAYER TRANSPORT AND MIXING FOR Estimating Urban-Scale GreenhOUSE Gas EMisSiONS
}

\author{
R. Michael Hardesty ${ }^{l}$, W. Alan Brewer ${ }^{2}$, Scott P. Sandberg ${ }^{2}$, Ann M. Weickmann ${ }^{1}$, Paul B. \\ Shepson $^{3}$, Maria Cambaliza ${ }^{3}$, Alexie Heimburger ${ }^{3}$, Kenneth J. Davis ${ }^{4}$, Thomas Lauvaux ${ }^{4}$, Natasha \\ L. Miles ${ }^{4}$, Daniel P. Sarmiento ${ }^{4}$, A. J. Deng ${ }^{4}$, Brian Gaudet ${ }^{4}$, Anna Karion ${ }^{l}$, Colm Sweeney ${ }^{1}$, James \\ Whetstone $^{5}$ \\ ${ }^{I}$ Cooperative Institute for Research in Environmental Sciences, University of Colorado/NOAA, Boulder \\ CO, 80305 USA, *Email: mike.hardesty@noaa.gov \\ ${ }^{2}$ NOAA Earth System Research Laboratory, CSD, Boulder, CO 80305 \\ ${ }^{3}$ Purdue University, Lafayette, IN USA \\ ${ }^{4}$ Pennsylvania State University ${ }^{3}$, College Station, PA USA \\ ${ }^{5}$ National Institute of Standards and Technology ${ }^{7}$ Gaithersburg, MD, USA
}

\begin{abstract}
A compact commercial Doppler lidar has been deployed in Indianapolis for two years to measure wind profiles and mixing layer properties as part of project to improve greenhouse measurements from large area sources. The lidar uses vertical velocity variance and aerosol structure to measure mixing layer depth. Comparisons with aircraft and the NOAA HRDL lidar generally indicate good performance, although sensitivity might be an issue under low aerosol conditions.
\end{abstract}

\section{INTRODUCTION}

The Indianapolis Flux Experiment (INFLUX) is aimed at improving methods for estimation of greenhouse gas emissions at urban scales. Current estimates rely on "bottoms-up" techniques, in which emissions from individual sources within the area of interest are summed to produce an overall estimate. The INFLUX experiment incorporates aircraft and surface-based observations, as well as chemical transport models, to investigate potential improvement in regional scale emissions gained by applying a topdown approach. INFLUX observational components include several-times-per-month aircraft measurements of gas concentrations and meteorological parameters, as well as a number of towers observing $\mathrm{CO}_{2}, \mathrm{CH}_{4}$, and $\mathrm{CO}$ and a single continuously operating Doppler lidar to estimate wind, turbulence and aerosol structure in the boundary layer. Here we discuss the role and utility of Doppler lidar observations to characterize the boundary layer during INFLUX.

\section{ROLE OF DOPPLER LIDAR IN INFLUX}

INFLUX focuses on developing and assessing model and observational techniques for estimating greenhouse gas emissions. An important element is the application of mass balance techniques [1] in which an aircraft equipped with chemical sensors samples greenhouse gas concentrations upwind and downwind of the city, as shone in Fig. 1. The Doppler lidar is a critical element, providing the needed estimates of wind speed and direction, boundary layer depth, and mixing needed for calculation of the upwind and downwind flux, from which the emission rates can be computed.

\section{INDIANAPOLIS DEPLOYMENT}

Utility of Doppler lidar for mass balance studies was demonstrated during the Uintah ozone study [1] where the NOAA High Resolution Doppler Lidar (HRDL, [2]) was used in conjunction with aircraft measurements to assess leakage from gas wells in the Uintah basin oil and gas field. Because INFLUX was designed to be a multi-year study, we opted to deploy a commercial, lowpulse-energy, high pulse rate lidar for the study [3]. The lidar, purchased from Halo Photonics, was deployed on the roof of a classroom building at Ivy Tech Community College northeast of 


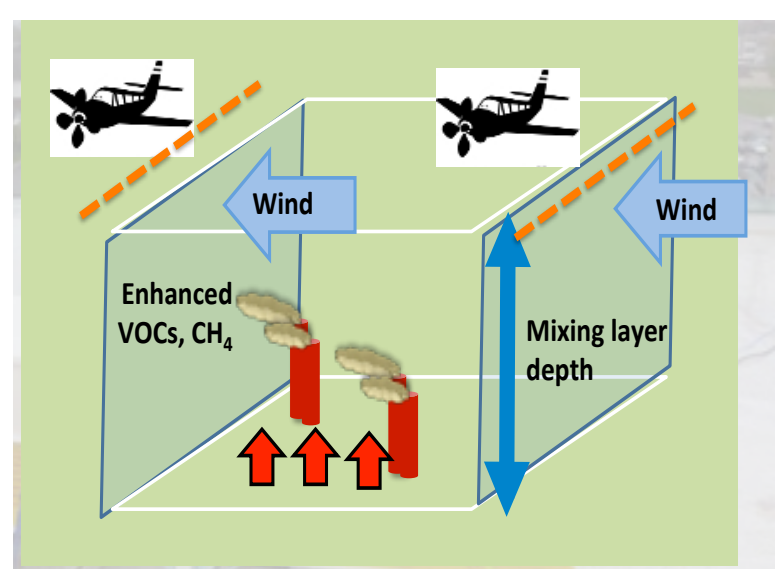

Figure 1: Schematic showing mass-balance technique for estimating emissions.

Indianapolis in April, 2013, and operated, with the exception of a maintenance interval in summer 2013, continuously until spring, 2015, when it was sent back to the manufacturer for maintenance and performance upgrades. A fixed scan pattern, repeated every 20-30 minutes, was implemented to measure the needed boundary layer parameters. The pattern is comprised of several conical scans at different elevation angles for measurement of horizontal wind profiles, vertical scans at orthogonal azimuths for observations of low level winds under stable conditions, and fixed vertical stares to observe vertical turbulence and mixing. Estimates of vertical velocity variance and aerosol backscatter profiles are computed from the vertical stares to characterize atmospheric mixing.

Figure 2 shows a 12-hour time height crosssection of vertical velocity variance and aerosol backscattered signal observed during the daytime (12Z-00Z) on September 13, 2013. The growth of the mixing layer to about $1500 \mathrm{~m}$ is clearly seen in both figures. However, the presence of a residual aerosol layer extending from the surface to $\sim 1 \mathrm{~km}$ above the surface between $12 \mathrm{Z}$ and $16 \mathrm{Z}$ is also observed, complicating the ability to measure mixing layer depth during that period. For this reason we use both aerosol and vertical velocity variance for estimation of mixing layer depth.

The vertical velocity variance profile in Fig. 2 also shows evidence of uncertainties due to sampling error. Because staring is typically carried out for 10-15 minutes of every scan sequence, the inability to statistically represent

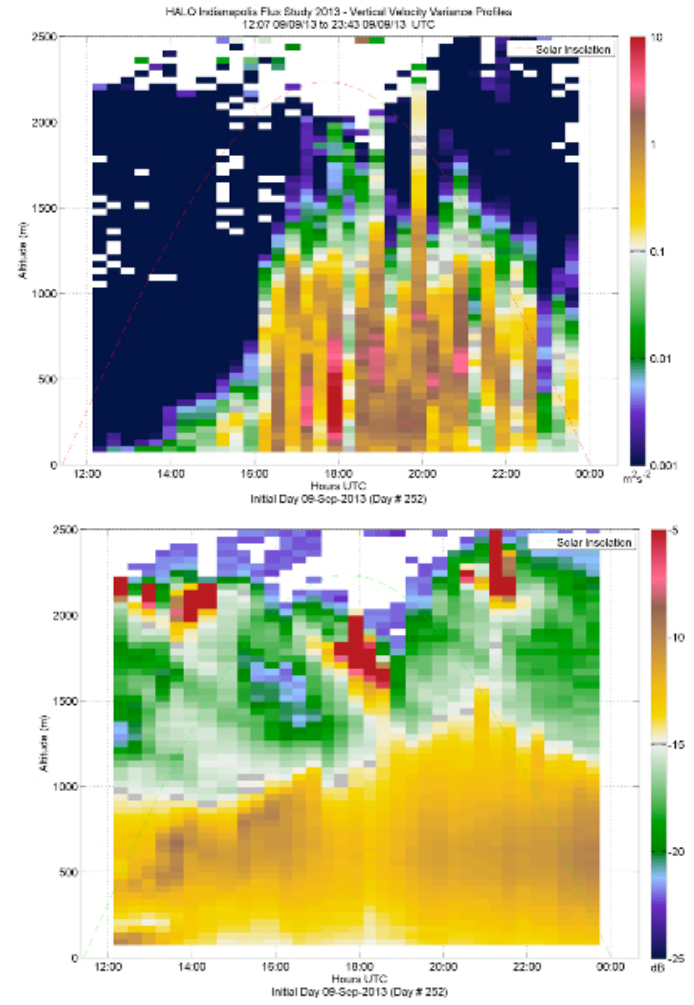

Figure 2: Time-height cross-sections showing vertical velocity variance (top) and aerosol signal (bottom) over a 12 hour daytime period.

large eddies under certain conditions is manifested as variability in the estimated mixing height.

Figure 3 shows wind profiles computed from conical and vertical scans from the same period. Note that this scanning strategy, which includes several low elevation scans, provides highresolution measurements near the surface where

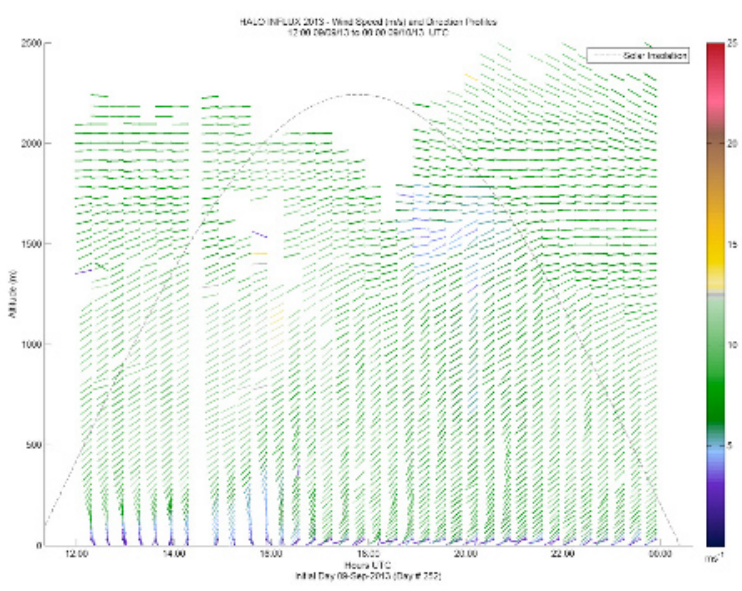

Figure 3: 12-hour time-height cross section of wind profiles computed from lidar conical scans. 
wind structure can be quite detailed.

\section{TWO-LIDAR OBSERVATION PERIOD}

During spring 2014 we deployed our HRDL lidar in Indianapolis for comparison studies with the INFLUX Halo instrument and to investigate spatial variability of the lidar-measured properties. For studying spatial variability, the HRDL was situated at Indianapolis International Airport, southwest of the city. Measurements of boundary layer properties were obtained by both lidars in conjunction with mass-balance studies incorporating observations from the Purdue aircraft ([4] The flights typically included spirals above the lidar site(s), thus providing opportunities to compare aircraft and lidar estimates of boundary layer properties.

Figure 4 shows lidar measurements of vertical velocity variance, along with aircraft measurements of $\mathrm{CO}_{2}$, methane, potential temperature and water vapor observed during aircraft spirals over the lidar sites at the airport and Ivy Tech. The spirals are displaced in time by slightly less than one hour. From the figures, it can be seen that the mixing layer is growing steadily during this period. Mixing layer depths at the Ivy Tech site observed by the lidar are deeper than those observed at the airport- this is also seen in the aircraft data. It is worth noting that estimating the mixing layer depth from the aircraft observations in this case is not straightforward the profiles of both the greenhouse gases and meteorological variables show significant structure, perhaps because the aircraft is sampling within several distinct convective plumes. Over the course of the 2-year deployment a number of similar aircraft-lidar comparisons exist and are being analyzed to assess different techniques for estimating mixing layer depth as it pertains to mass-flux measurements.

The lidar observations are also of significant use in assessing and improving the performance of chemical models to estimate emissions. Figure 5 shows the improvement obtained by assimilating 12 hours of lidar wind data into a WRF chemical model. The model readily accepts the lidar data

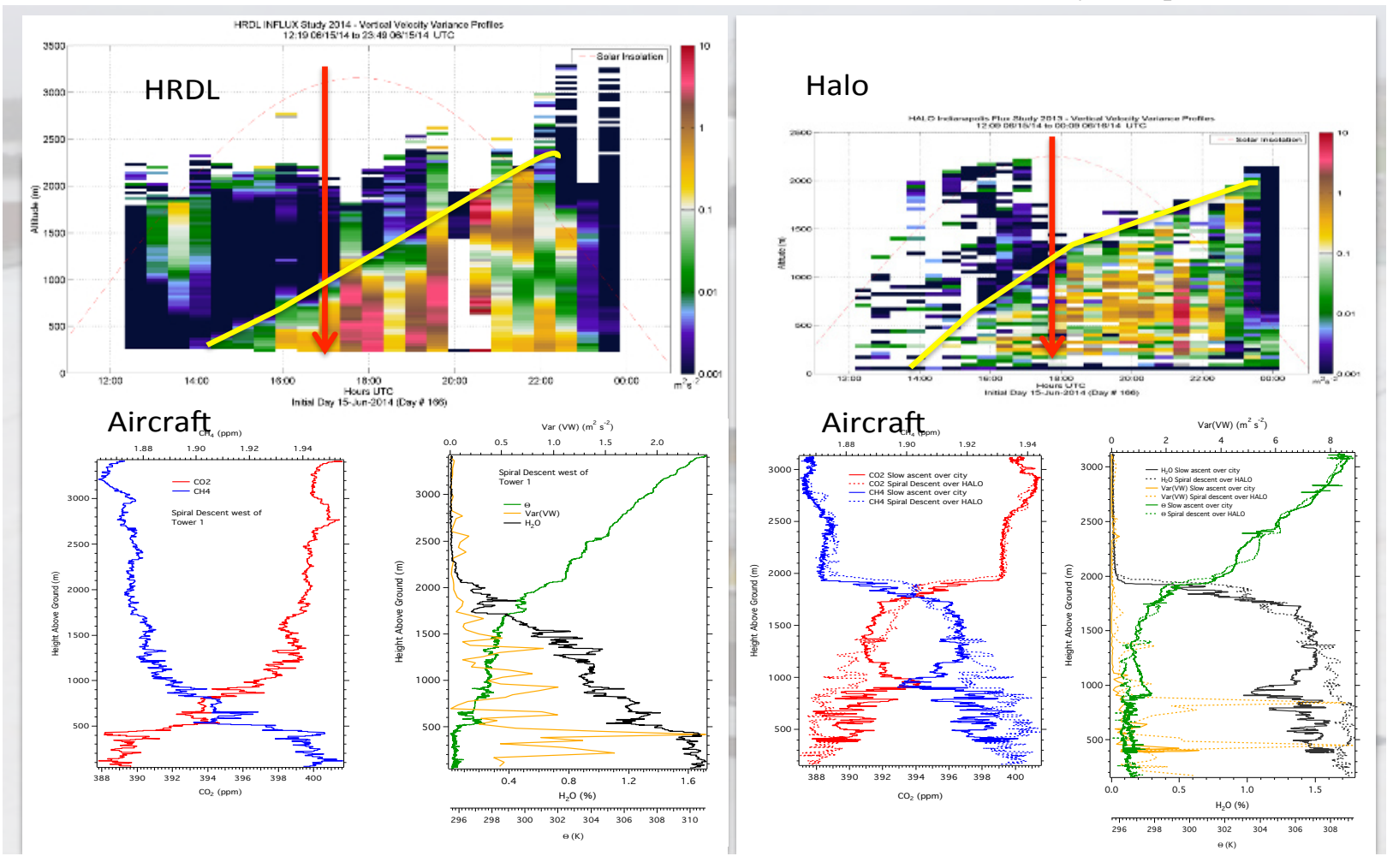

Figure 4: Observations of vertical velocity variance from HRDL (top, left) and Halo (top, right) compared with aircraft in situ sampling (bottom) of CO2 (red), methane (blue,) water vapor (black) and potential temperature (green). 


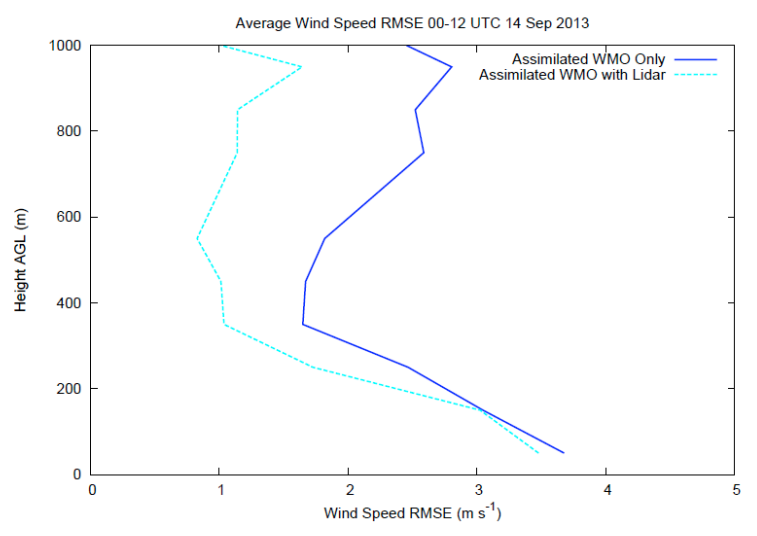

Figure 5: Improvement in model estimates of wind profile based on assimilation of lidar wind profiles during INFLUX.

and uses it to improve the wind estimate, indirectly verifying the utility of the observations.

\section{INVESTIGATING SENSITIVITY}

A key requirement for a lidar deployed for longterm measurements of boundary layer properties is the ability to reach the top of the mixing layer under a variety of conditions. During periods of low aerosol loading, we have seen indications that, unlike HRDL, the Halo instrument does not always see the full mixing layer. We have compared measurements from the HRDL and Halo instruments in side-by-side comparisons. Generally, it is seen that the measurements from the Halo instrument are noisier than those from HRDL, which is not unexpected given the two order-of-magnitude difference in pulse energy. HRDL always sees the terrestrial mixed layer and typically penetrates further into the free troposphere. It is also observed that the direct wind speed comparisons are generally in good agreement. We are currently testing algorithms to determine how often the Halo instrument is not seeing the full boundary layer in order to assess whether this is significant for future deployments.

\section{FUTURE PLANS}

We are continuing deployment of the Halo lidar at INFLUX. However, the lidar is scheduled to be returned to the manufacturer in spring 2015 for modification to improve sensitivity. Prior to redeployment in Indianapolis, the instrument will be installed next to HRDL in Boulder for additional sensitivity tests. We also intend to include a micro-pulse aerosol lidar for studying the relative capability to estimate mixing layer depth.

\section{ACKNOWLEDGEMENTS}

We appreciate the assistance of INFLUX colleagues Jocelyn Turnbull and Kevin Gurney in providing insights on greenhouse gas emissions. This research was partially supported by the National Institute of Standards and Technology.

\section{REFERENCES}

[1] Petron, G., A. Karion, C. Sweeney, et al, 2014: A new look at methane and nonmethane hydrocarbon emissions from oil and natural gas operations in the Colorado Denver-Julesburg Basin, J. Geophys. Res., 119, 6836-6852.

[2] Grund, C. J.,R. M. Banta, J. L. George et al, 2001: High resolution Doppler lidar for boundary layer and cloud research, J. Atmos. Ocean. Tech., 18, 376-393.

[3] Hogan, R. J., A. L. Grant, A. J. Illingworth, et al., 2009: Vertical velocity variance and skewness in clear and cloud-topped boundary layers as revealed by Doppler lidar. Quart. J. Roy. Meteor. Soc., 135, 635-643.

[4] Cambaliza, M. O. L., P. B. Shepson, D. R. Caulton, et al, 2014: Assessment of uncertainties of an aircraft-based mass balance approach for quantifying urban greenhouse gas emissions, Atmos. Chem. Phys., 14, 9029-9050. 